\title{
Antimicrobial Activity of Aqueous Plant Extracts as Potential Natural Additives ${ }^{\dagger}$
}

\author{
Mariana C. Pedrosa 1, Jonata M. Ueda 1, Sandrina Heleno 1, Bruno Melgar 1, Marija Ivanov ${ }^{2}$, Marina Soković ${ }^{2}$, \\ Marcio Carocho ${ }^{1, *}$, Isabel C. F. R. Ferreira ${ }^{1}$ and Lillian Barros ${ }^{1, *}$
}

1 Centro de Investigação de Montanha (CIMO), Instituto Politécnico de Bragança, Campus de Santa Apolónia, 5300-253 Bragança, Portugal; marianapedrosa@ipb.pt (M.C.P.); massaoueda@hotmail.com (J.M.U.); sheleno@ipb.pt (S.H.); bruno.melgarc@ipb.pt (B.M.); iferreira@ipb.pt (I.C.F.R.F.)

2 Institute for Biological Research "Siniša Stancović" - National Institute of the Republic of Serbia, University of Belgrade, Bulevar Despota Stefana 142, 11000 Belgrade, Serbia; marija.smilijkovic@ibiss.bg.ac.rs (M.I.); mris@ibiss.gb.ac.rs (M.S.)

* Correspondence: mcarocho@ipb.pt (M.C.); lillian@ipb.pt (L.B.)

+ Presented at the 1st International Electronic Conference on Food Science and Functional Foods, 10-25 November 2020; Available online: https://foods_2020.sciforum.net/.

Citation: Pedrosa, M.C.; Ueda, J.M. Heleno, S.; Melgar, B.; Ivanov, M.; Soković, M.; Carocho, M.; Ferreira, I.C.F.R.; Barros, L. Antimicrobial Activity of Aqueous Plant Extracts as Potential Natural Additives. Proceedings 2020, 70, 79. https:// doi.org/10.3390/foods_2020-07595

Published: 9 November 2020

Publisher's Note: MDPI stays neutral with regard to jurisdictional claims in published maps and institutional affiliations.

Copyright: $₫ 2020$ by the authors. Licensee MDPI, Basel, Switzerland. This article is an open access article distributed under the terms and conditions of the Creative Commons Attribution (CC BY) license (http://creativecommons.org/licenses/by/4.0/).

\begin{abstract}
The 21st century has brought along many changes in how consumers look at food and perceive their diets. There is an increasing awareness towards what goes into manufacturing these foods, with an important concern being drawn towards food additives. While it is known that additives are needed to preserve or change attributes of food, it is also widely understood that consumers prefer natural additives to artificial ones, and thus, the industry is looking for alternatives from plant sources. The extraction of five different plants (oregano (Origanum vulgare L.), rosemary (Rosmarinus officinalis L.), salvia (Salvia officinalis L.), lemon balm (Melissa officinalis L.), and basil (Ocimum basilicum L.)) was optimized for three extraction types, namely decoction, infusion, and cold hydroethanolic (80:20) ultrasound-assisted extraction. This optimization was carried out through a screening analysis to find the most important factors, using Design Expert, pending the analysis of phenolic compounds through HPLC-DAD-ESI/MS. The optimized variables used were temperature, time of extraction, and potency (ultrasound). The extraction with the highest phenolic content for each plant was then selected and screened for its antibacterial and antifungal activity, relying on the microdilution method against foodborne pathogens. The bacterial strains used were Staphylococcus aureus, Bacillus cereus, Listeria monocytogenes, Escherichia coli, Salmonella typhimurium and Enterobacter cloacae, while the fungal strains were Aspergillus fumigatus, Aspergillus niger, Aspergillus versicolor, Penicillium funiculosum, Trichoderma viride, and Penicillium verrucosum var. cyclopium. Two synthetic and widely used preservatives, namely sodium benzoate (E211) and potassium metabisulfite (E224), were also screened against these contaminants to confirm the sensitivity of the microorganisms to these known artificial preservatives. Overall, the plant extracts showed a high inhibition of fungi, with all extracts showing lower minimum inhibition concentrations than both the synthetic preservatives, except for $P$. viridae, where E224 showed the same inhibition capacity. Regarding the antibacterial activity, the bacteria most sensitive to the extracts was $B$. cereus, for which all the extracts showed the same activity as E224. Lemon balm was the stronger extract, showing the same inhibition as E221 against B. cereus and E. cloacae. Overall, this work proves that plant extracts obtained though "green" and low-cost technologies can be alternatives to artificial food additives, due to showing the same, or, in some cases, better antimicrobial activity. Furthermore, a mixture of these extracts can result in synergistic effects and improve the antimicrobial activities. The next step of this work, which is ongoing, will focus on the determination of the efficacy of these extracts and their incorporation in muffins.
\end{abstract}

Keywords: plant extracts; food additives; preservatives; response surface methodology 


\section{Introduction}

The shelves on supermarkets are stocked with fresh fruit, vegetables, meats, and fish from many provenances, and prepared meals, processed goods, and other food with high freshness and quality. All of these products have to endure the journey from harvest, to processing, to the store, and finally, to the consumer's house. Once bought, they must endure on the consumer's shelf for some more time. For foods to withstand the passage of time, the industry relies on food additives, which are now one of the most widespread means of food preservation. Still, the use of additives has not been consensual over the recent decades, with food scares and scandals involving these molecules. With the turn of the century, and mainly in the past 10 years, consumers have become more aware of the food they eat, and the consequences of this food on their health. The consequence of this has been a preference for unprocessed food, and, when processed, a clear preference for the use of natural food additives [1]. Overexposure to synthetic food additives has been related to health issues, and thus, the industry now turns to nature to use the molecules it has to offer, rather than using them as an inspiration for various purposes. In this scenario, provided that the preservation of food is one of the most important tasks, the use of plant extracts has been increasing to carry it out. Specifically, rosemary extract is one of the most relevant examples, being approved by the European Food Safety Authority to be used in various foods. Other extracts have been used successfully although they have not yet been approved [2,3]. Using plants as preserving agents could be a game changer in the food industry by allowing by-products of crop production to be used as sources for these additives, contributing to a sustainable production and reducing agricultural waste.

\section{Materials and Methods}

\subsection{Reagents and Microorganisms}

The Gram-positive bacteria Staphylococcus aureus (ATCC 11632), Bacillus cereus (clinical isolate), Listeria monocytogenes (NCTC 7973), as well as the Gram-negative bacteria Escherichia coli (ATCC 25922), Salmonella Typhimurium (ATCC 13311), and Enterobacter cloacae (ATCC 35030) were used in order to determine the antibacterial activity of the samples. For antifungal assays, six micromycetes were used: Aspergillus fumigatus (human isolate), Aspergillus niger (ATCC 6275), Aspergillus versicolor (ATCC11730), Penicillium funiculosum (ATCC 36839), Trichoderma viride (IAM 5061), and Penicillium verrucosum var. cyclopium (food isolate). Dimethyl sulfoxide, p-iodononitrotetrazolium violet, sodium benzoate (E211), and potassium metabisulfite (E224) were acquired from Sigma-Aldrich (Sigma-Aldrich, St. Louis, MO, USA).

\subsection{Plant Material}

The plants used in this work - oregano (Origanum vulgare L.), rosemary (Rosmarinus officinalis L.), salvia (Salvia officinalis L.), lemon balm (Melissa officinalis L.), and basil (Ocimum basilicum L.) - were all acquired from the enterprise Cantinho das Aromáticas in Portugal, having arrived at the lab in a dried form. They were subsequently ground to a 20mesh powder.

\subsection{Ultrasound-Assisted Extraction (UAE)}

Ultrasound-assisted extraction of bioactive compounds from the different species analyzed was performed using an ultrasonic system (QSonica, model CL-334, Newtown, Connecticut, EE. UU). The solid-to-liquid ratio was adjusted in every repetition, maintaining a constant volume between every run, and right after each extraction, samples were centrifuged $\left(5000 \mathrm{rpm}\right.$ for $20 \mathrm{~min}$ at $10^{\circ} \mathrm{C}$ ), filtered through filter paper (Whatman $\mathrm{n}^{\circ} 4$ ), and dried at $40^{\circ} \mathrm{C}$ using a vacuum rotary evaporator (Büchi R-210, Flawil, Switzerland). The aqueous fraction was then lyophilized $\left(47^{\circ} \mathrm{C}, 0.045\right.$ bar; FreeZone 4.5, Labconco, Kansas City, MO, USA) to obtain the powdered extract. The extracts were stored in a dry place prior to further analysis. This design was built and randomized using Design Expert 
12.0.1. (Stat-Ease, Inc. Minneapolis, MN, USA. The fixed variables in this experiment were defined as X1: time (7.5 and $12.5 \mathrm{~min}), \mathrm{X} 2$ : solvent ( 0 and $80 \% \mathrm{EtOH})$, and X3: UAE Power (275 and $450 \mathrm{~W}$ ). The dependent variable was expressed according to the Rosmarinic acid content $(\mathrm{Y} 1)$. In the fractional factorial design, factors were aliased as Intercept $=$ Intercept $+\mathrm{ABC} ; \mathrm{A}=\mathrm{A}+\mathrm{BC} ; \mathrm{B}=\mathrm{B}+\mathrm{AC}$; and $\mathrm{C}=\mathrm{C}+\mathrm{AB}$, in order to determine pertinent factors and ranges of experimentation to be used in future optimization designs. Secondary selection and evaluation of factors and levels were carried out to determine the appropriate experimental domains following the guidelines of the first trial. Independent variables included X1: time (10-300 s) and X4: temperature $\left(20-75^{\circ} \mathrm{C}\right)$ at 6 and 4 different levels, respectively. For each of the 2 factors, a unifactorial multilevel or general factorial was performed and analyzed.

\subsection{Phenolic Fingerprinting and Quantification, and Statistical Analysis}

The chromatographic data was acquired using a Dionex Ultimate 3000 UPLC (Thermo Scientific, San Jose, CA, USA) coupled to a diode array detector (280, 330, and $370 \mathrm{~nm}$ ) and an electrospray ionization mass detector (Linear Ion Trap LTQ XL, Thermo Finnigan, San Jose, CA, USA), working in negative mode. The chromatographic separation was performed using a Waters Spherisorb S3 ODS-2 C18 $(3 \mu \mathrm{m}, 4.6 \mathrm{~mm} \times 150 \mathrm{~mm}$, Waters, Milford, MA, USA) column at $35^{\circ} \mathrm{C}$. The compounds were identified considering the retention time, $\mathrm{UV}-\mathrm{Vis}$, and mass spectra in comparison with available standards and with literature data. For the fractional factorial design, Pareto and analysis of variance (ANOVA) was carried out to determine the principal effects and their magnitudes, while, for the general factorial designs, comparison of different samples was carried out through the application of one-way analysis of variance (ANOVA) using an F-test with $p$-value = 0.05 , along with the least significant difference (LSD) Fischer's test. Both analyses were performed using Statgraphics Centurion XVI software (StatPoint Technologies, Inc. Warrenton, VA, USA).

\subsection{Antimicrobial Activity}

The extracts were dissolved in 5\% DMSO and screened against the microorganisms listed above. These microorganisms were obtained from the mycological laboratory of the Institute for Biological Research "Sinisa Stanković" in Serbia. Minimum inhibitory concentrations (MIC) were determined by a serial microdilution method, and the rapid $p$ iodonitrotetrazolium violet (INT) colorimetric assay, following the protocol described by Soković et al. (2010) and Soković and van Griensven (2006) [4]. For the negative control, $5 \%$ DMSO was used, while positive controls were streptomycin and ampicillin for the antibacterial activity, and ketoconazole and bifonazole for the antifungal activity, with the results expressed as MICs, MBCs, and MFCs in $\mathrm{mg} / \mathrm{mL}$.

\section{Results and Discussion}

\subsection{Extraction Optimization Studies}

Factor selection had been completed on previous optimization design, performed within the research group and available literature in order to reduce time and maximize yields. The choice for using UAE was highlighted; therefore, the most relevant factors to include were the time of extraction, potency, and percentages of ethanol and water used as an extraction solvent. A decision was made to work with the lowest UAE power in order to save resources, as well as with a high ethanol concentration in the extraction solvent. The efficiency of extraction with less polar solvents on the yielding of rosemary phenolic compounds has been also described by other authors (Oliveira et al., 2016; Sik et al., 2020), which reinforce this decision [5,6]. Regarding Figure 1a, it is clear that lemon balm is the plant which yields not only more phenolic compounds, but also the highest content of rosmarinic acid. In total, four peaks were positively identified by comparison with com- 
mercial standards, taking into account their retention time, mass, and UV-vis characteristics. Peak 1 presented a pseudomolecular ion $[\mathrm{M}-\mathrm{H}]^{-}$at $\mathrm{m} / \mathrm{z} 421$ and was tentatively identified as 4-Hydroxy-7-O-(3'-hydroxy-4'-O-glucosylbenzoyl) benzyl alcohol. Peaks 2 and 4 were identified as luteolin-O-glucuronide and kaempherol-O-glucuronide, respectively, both presenting a pseudomolecular ion $[\mathrm{M}-\mathrm{H}]^{-}$at $\mathrm{m} / \mathrm{z} 461$. Finally, Peak 3 was the major compound found in the plants and presented a pseudomolecular ion $[\mathrm{M}-\mathrm{H}]^{-}$at $\mathrm{m} / \mathrm{z} 359$, identified as rosmarinic acid.
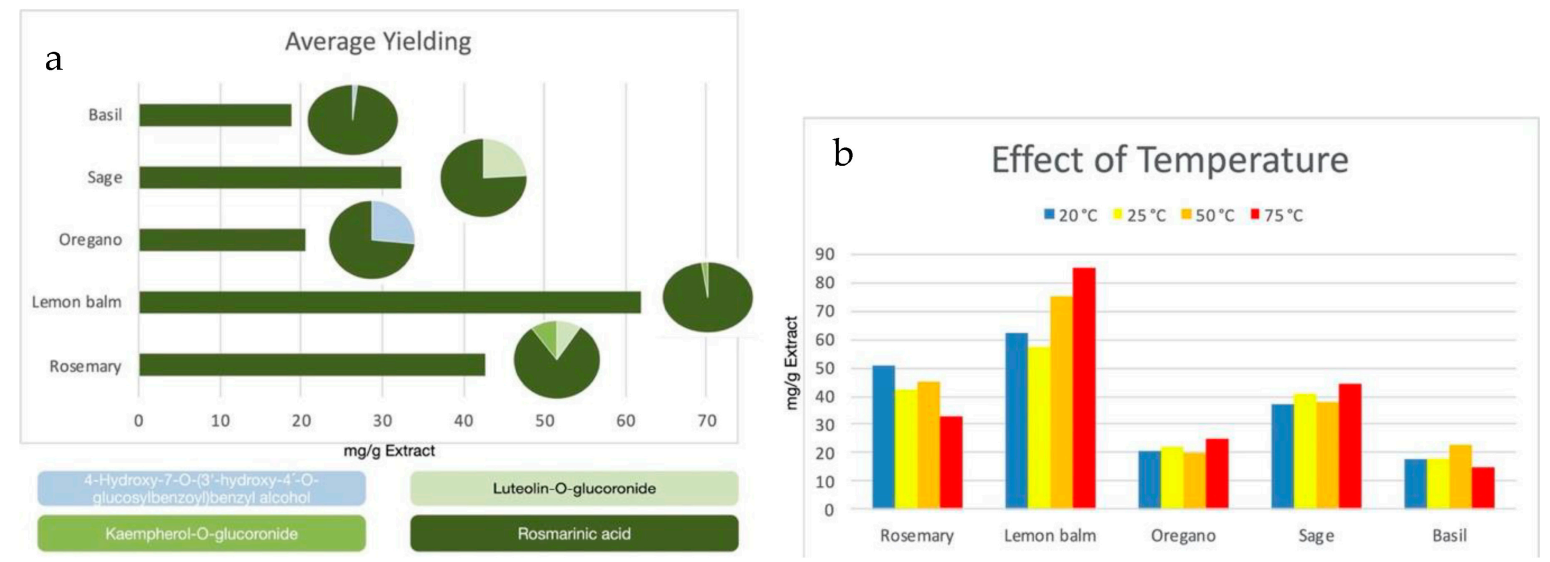

Figure 1. (a) Representation of the yield of each plant in terms of the total of the different phenolic compounds; (b) effect of temperature of the extractability of the phenolics.

In Figure $1 b$, the effect of temperature is shown for all plants, plotting it against the amount of extract obtained from the UAE extractions. Interestingly, the behavior of each plant was distinct. Rosemary showed a decrease of phenolic content with the increase of heat, while lemon balm increased the quantity of polyphenols with the increase of heat. The yields of sage, basil, and oregano were not affected by the variation in temperature. Overall, considering the cost of heating water at an industrial level, it does not seem be significantly beneficial to use heat to extract compounds from these plants with ultrasounds.

Figure 2 shows the variation of extractive capacity of the two plants with the highest amounts of total phenolic compounds for the different extraction times, namely 10, 15, and $30 \mathrm{~s}$, and 1, 2, and $5 \mathrm{~min}$. Considering rosemary (Figure 2a), it is quite clear that extending the extraction time did not significantly increase the yield in phenolic compounds. For instance, $10 \mathrm{~s}$ yielded almost the same amount of total phenolic compounds as $5 \mathrm{~min}$. A very similar effect was seen for lemon balm (Figure $2 b$ ), in which there was a higher amount of total phenolic compounds for 2 and 5 min. Considering the cost of operation and the extraction time, the yield of $10 \mathrm{~s}$ seems to be quite interesting, especially due to the very small variation in total phenolics when compared to the other times.
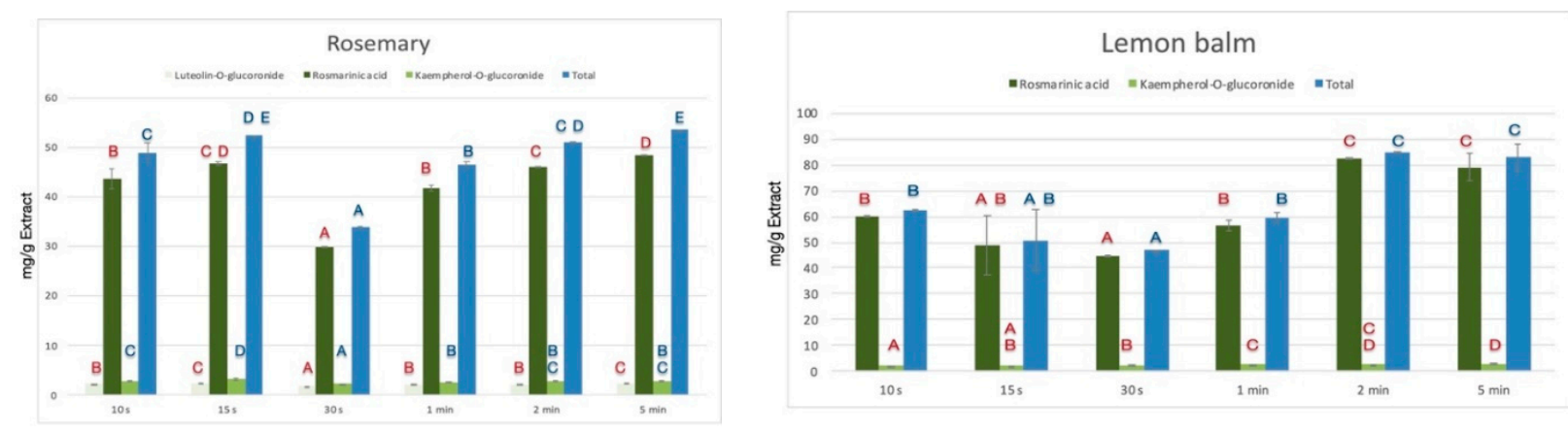

Figure 2. Representation of the variation of rosemary and lemon balms in terms of time of extraction. 


\subsection{Antimicrobial Analysis}

In Figure 2, the antimicrobial analysis is represented, with the positive controls on the right of each group of results in dark grey and black, the synthetic preservatives in light green and cyan, and the plant extracts in darker colors. In Figure 3a, the MIC of the foodborne bacteria are shown. None of the plant extracts of the synthetic compounds showed values as low as the positive controls, although rosemary and salvia did show some promising effects, namely being better than or having an equal effect as sodium benzoate for all bacteria except Bacillus cereus. A very similar effect was sought for MBC (Figure 3b), where these two plant extracts showed similar effects to sodium benzoate in killing the bacteria. Still, overall, potassium metabisulfite proved to be very effective against all but Bacillus cereus. Concerning the fungi, Figure 3c shows the MICs. Overall, the plant extracts seem to have a higher effect against fungi than bacteria. All plant extracts showed better antimicrobial activity than both synthetic preservatives; in fact, the values of the plant extracts were very close to the positive controls. Specifically, for $A$. fumigatus, $P$. funiculosum, and $P$. verrucosum, salvia and rosemary showed values under or equal to the positive controls, revealing an excellent capacity to be used in food to hinder fungi growth. The same tendency is seen for MFC (Figure 3d), where the extracts have lower values for all fungi when compared to sodium benzoate, but also better than potassium metabisulfite for, once again, A. fumigatus, P. funiculosum, and P. verrucosum.

\section{Conclusions}

In conclusion, UAE seems to be a promising method to obtain polyphenolic extracts from Lamiaceae plants, namely rosemary, lemon balm, sage, oregano, and basil. Rosemary and basil showed the highest amounts of polyphenols of the studied plants. UAE is a very easy and rapid method to obtain these plant extracts, at any temperature and in brief bursts of time. The obtained extracts could be used as natural antimicrobials for foods, due to their interesting antimicrobial activity, comparable in some instances to commercial artificial counterparts. 


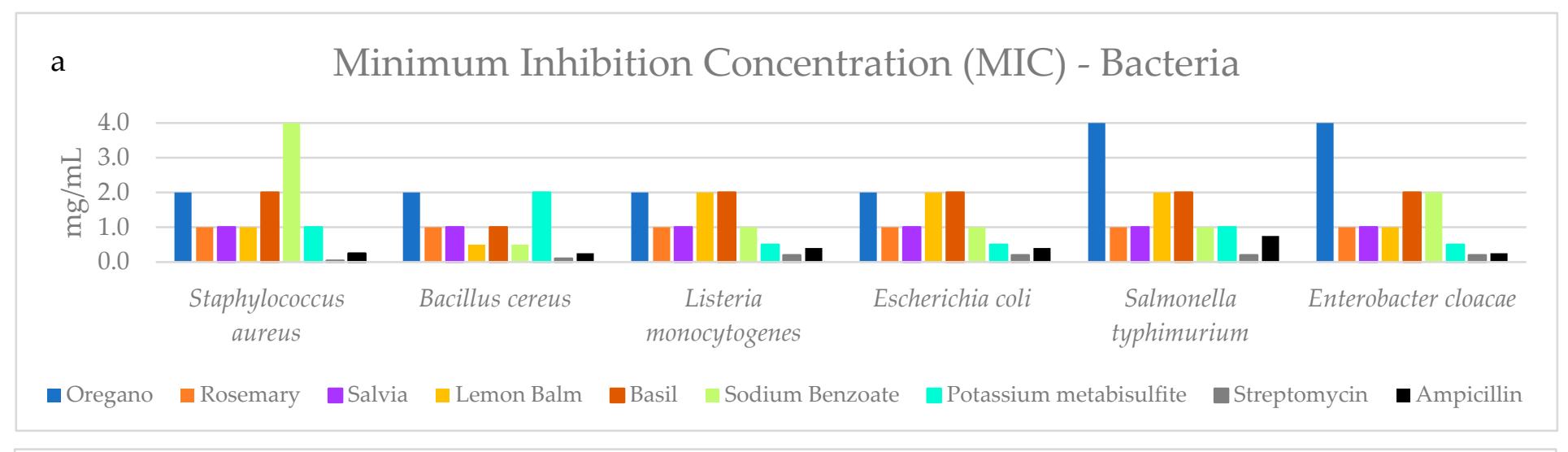

b Minimum Bactericidal Concentration (MBC)

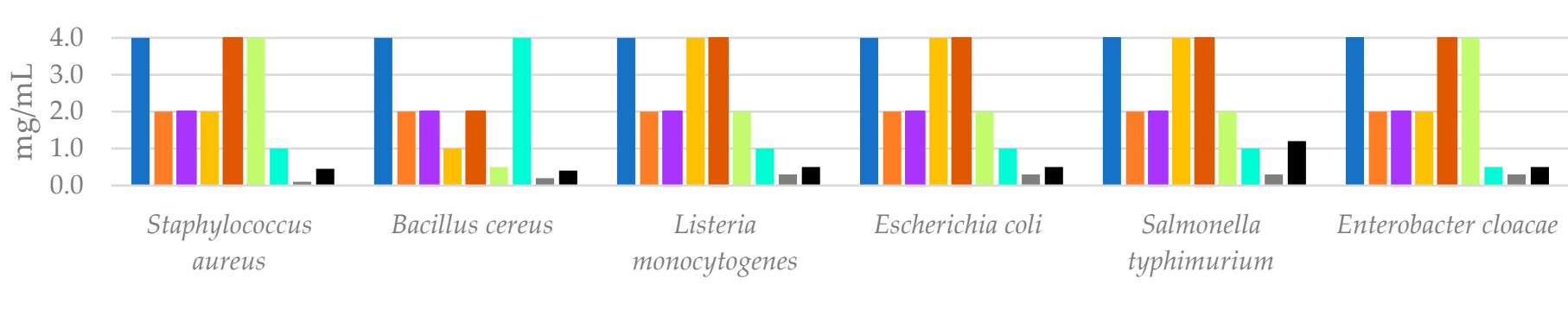

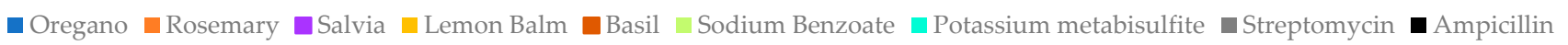




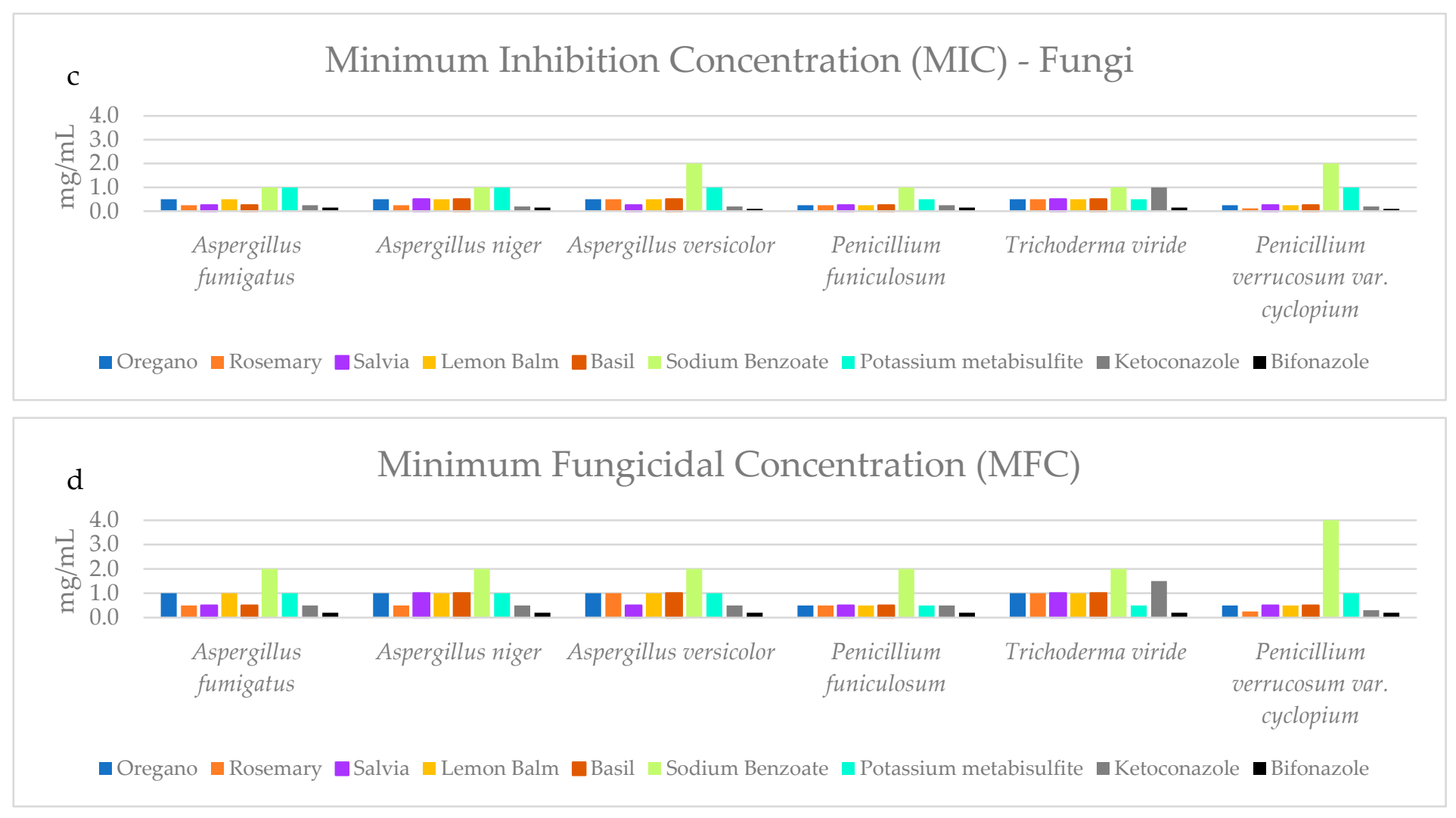

Figure 3. Charts representing (a) MIC of antibacterial activity, (b) MBC, (c) MIC of antifungal activity, (d) MFC. 
Author Contributions: Conceptualization, M.C. and S.H.; methodology, M.C.P., J.M.U., and M.I.; software, B.M.; validation, M.C.; investigation, M.C.P. and J.M.U.; data curation, B.M.; writingoriginal draft preparation, M.C and B.M; supervision, M.S., L.B., and I.C.F.R.F.; project administration, L.B. All authors have read and agreed to the published version of the manuscript.

Funding: This research was funded by the Portuguese Foundation for Science and Technology (FCT, Portugal) through national funds FCT/MCTES to CIMO (UIDB/00690/2020). S. Heleno and M. Carocho thank FCT for their individual research program-contract (CEECIND/00831/2018; CEECIND/03040/2017), while L. Barros thanks FCT for their institutional scientific employment programcontract. This work was also funded by the European Regional Development Fund (ERDF), through the Incentive System to Research and Technological development, within the Portugal2020 Competitiveness and Internationalization Operational Program within the scope of Project Mobilizador, POCI-01-0247-FEDER-046112: BIOMA.

Institutional Review Board Statement: Not applicable.

Informed Consent Statement: Not applicable.

Data Availability Statement: Data available on request.

Conflicts of Interest: The authors declare no conflict of interest.

\section{References}

1. Carocho, M.; Morales, P.; Ferreira, I.C.F.R. Natural food additives: Quo vadis? Trends Food Sci. Technol. 2015, 45, $284-295$.

2. Conceição, N.; Albuquerque, B.R.; Pereira, C.; Corrêa, R.C.G.; Lopes, C.B.; Calhelha, R.C.; Alves, M.J.; Berros, L.; Ferreira, I.C.F.R. By-products of camu-camu [Myrciaria dúbia (Kunth) McVaugh] as promising sources of bioactive high added-value food ingredients: Functionalization of yogurts. Molecules 2020, 25, 70.

3. Veloso, F.S.; Caleja, C.; Calhelha, R.C.; Pires, T.C.S.; Alves, M.J.; Barros, L.; Genena, A.K.; Barreira, J.C.M.; Ferreira, I.C.F.R. Characterization and application of pomegranate epicarp extracts as functional ingredients in a typical Brazilian pastry product. Molecules 2020, 25, 1481.

4. Soković, M.; Glamočlija, J.; Marin, P.D.; Brkić, D.; Griensven, J.L.D. Antibacterial effects of the essential oils of commonly consumed medicinal herbs using an in vitro model. Molecules 2010, 15, 7532-7546.

5. Oliveira, G.D.A.R.; Oliveira, A.E.; Conceição, E.C.; leles, M.I.G. Mutiresponse optimization of na extraction procedure of carnosol and rosmarinic and carnosic acids from rosemary. Food Chem. 2016, 211, 465-473.

6. Sik, B.; Hanczné, E.L.; Kapcsándi, V.; Ajtony, Z. Conventional and nonconventional extraction techniques for optimal extraction processes of rosmarinic acid and six Lamiaceae plants as determined by HPLC-DAD measurement. J. Pharm. Biomed. Anal. 2020, $184,113173$. 